\title{
“Let Me Tell You Why!". When Argumentation in Doctor-Patient Interaction Makes a Difference
}

\author{
SARA RUBINELLI*, PETER J. SCHULZ \\ Health Care Communication Laboratory, \\ School of Communication Sciences \\ University of Lugano \\ Via Buffi 13, 6900 Lugano \\ Switzerland \\ *E-mail: sara.rubinelli@lu.unisi.ch
}

\begin{abstract}
This paper throws some light on the nature of argumentation, its use and advantages, within the setting of doctor-patient interaction. It claims that argumentation can be used by doctors to offer patients reasons that work as ontological conditions for enhancing the decision making process, as well as to preserve the institutional nature of their relationship with patients. In support of these claims, selected arguments from real-life interactions are presented in the second part of the paper, and analysed by means of a model of argumentation borrowed from classical rhetoric, and refined according to the modern orientation of the pragma-dialectic approach.
\end{abstract}

KEY WORDS: doctor-patient relationship, pragma-dialectic theory of argumentation, ancient rhetoric, informed consent, decision-making

\section{INTRODUCTION}

In Plato's time argumentation was already linked to physicians. ${ }^{1}$ Today, however, speaking about argumentation in doctor-patient interaction provokes some concern and disagreement. As we have personally experienced in delivering papers on the subject, there is a general conviction that doctors, if they use argumentation, exercise power, influence and diminish patients' ability to choose for a treatment freely. As Dickinson (1998, pp. 74-75) claims, argumentation tends to be associated with eristic (and, we add, fallacious) dialogues. That doctors should not influence patients in choosing one treatment option instead of another and, thus, that they should not use argumentation, is clearly stated by Eddy (1990). We believe that most of these concerns result from preconceptions linked to the idea that argumentation falls under the instruments of a rather sophistic rhetoric. In answer to these preconceptions, it must be noted that from the time of Aristotle onward, argumentation has been explored by schools of prominent scholars as an instrument of 
intellectual refinement for enhancing efficacy in human communication. To quote a recent case among the most relevant explorations, the pragma-dialectic approach of the Amsterdam school emblematically shows how argumentation can indeed be structured to solve differences of opinions according to an ideal model of critical discussion. ${ }^{2}$

A few recent contributions tackle the issue of argumentation in medical encounters. ${ }^{3}$ Yet, to our knowledge and understanding, they do not seem to pay enough attention to the place that argumentation has in the doctor-patient relationship and, consequently, to its nature within the peculiarity of this interaction. This attention, however, is of fundamental importance to fully evaluate the potentialities that, we believe, argumentation has among the interactional tools at doctors' disposal. Without entering at this stage into the details of what argumentation is, it is sufficient to say that the process generally aims at convincing the listener or reader of the acceptability of a standpoint. Clearly, this occurs when the listener or reader explicitly, implicitly or potentially doubts the acceptability of the standpoint, or has a different standpoint. This aspect concerning the difference of opinions is precisely what makes argumentation in medical consultation so peculiar. Between a doctor and a patient, in fact, there is no initial conflict of standpoints. Both the doctor and the patient agree on the fact that the health of the latter must represent the final aim of the consultation itself. In this sense, argumentation only enters in a different stage of their interaction. Having anticipated this claim, this paper has two main aims. First, we intend to illustrate the place, implications and advantages that argumentation has in the doctor-patient consultation. In so doing, we shall lay down some essential bases for supporting the appropriateness of argumentation in this setting. Second, we shall make some analytical investigations of examples of real-life argumentations within the context of informed consent. This context is indeed intrinsically argumentative, insofar as doctors need patients' signatures before giving specific treatments. And signing the consent implies that the patient is convinced that the treatment suggested is what $\mathrm{s} / \mathrm{he}$ should do in that specific context. The present investigation is expected to underline some analytical aspects that it is important to consider in order to ground the study of argumentation in medical encounter on a more theoretical level and, ultimately, to enhance an optimisation of the argumentative practice in the field.

\section{THE PLACE OF ARGUMENTATION IN DOCTOR-PATIENT INTERACTION}

As already mentioned above, the doctor-patient interactional context is not traditionally perceived as argumentative. In the initial stage of the 
consultation both the doctor and the patient agree on the fundamental standpoint that the health of the patient, as final aim of the consultation, is all that really matters. Patients go to see their doctors essentially to ask for advice. It can be generalised that they normally lack any a priori attitude to argue with their doctors. While doctors possess the technical expertise on medical matter, patients, in the majority of cases, do not have a sufficient scientific or expert knowledge to effectively engage with doctors' suggestions on equal terms. In this sense, communication between doctors and patients implies an almost inevitable disparity of competence that ideally should prevent a real disagreement of points of view to arise on an issue. However, what seems a rather straightforward situation is clouded by a main factor that justifies doctors' use of argumentation in daily practice. In contrast to mathematics, that appears as a paradigm of precision and certainty, where certain moves lead to others predictable and expectable, in medicine the nature of the nexus cause-effects can neither be assured, nor predicted with certainty (Schulz, 2006). To lessen the consequent arbitrariness in the use of medication prescription and diagnostic testing, the new paradigm for de-emphasizing intuition in medical practice known as "evidence-based medicine" has been introduced. ${ }^{4}$

Yet, even if advice ultimately derives from a careful consideration of objective parameters, doctors are constantly in the position of having to make clinical decisions at different levels. In particular, what is relevant for the perspective of this paper, doctors are faced with the evidence that there are always more treatments (including the option of nontreatment) available for the same disease. And this means that, especially but not exclusively at the level of treatments, what doctors suggest results for the most part from their own choices among several possibilities for improving the quality of patients' lives. Even if medicine has always faced such limitations, in the past the subjective nature of doctors' advice would have not impacted on patients' compliance highly. Medicine was surrounded by the idea that the doctor, like the shaman, held the key to sickness and death. Nowadays, however, due to the amount of information available and the general influence that traditional and new media have on people's decisions, ${ }^{5}$ patients are becoming increasingly more aware of the risks linked to the choice of one treatment instead of another (Schulz, 2003). Physicians widely recognise that patients are becoming more and more critical about advice received: patients are inclined to gather information from sources other than their doctors, and to ponder what doctors suggest on the basis of their layknowledge. In this sense, provided that the doctor and the patient agree on the fact that the health of the latter represents the unique and final aim of all the efforts of the former, doctors are now in the need of legitimising their standpoints on what they see as the best way to treat the 
particular cases encountered. When the reliability of doctors' decisions is no longer taken for granted, argumentation begins to play a role. To address the potential risks linked to a dismissal of the doctor's authority - risks that especially concern the compliance of treatment from patients - this authority can be reinforced by adducing reasons in support of the doctors' points of view. Since medical knowledge risks invoking disagreement, doctors can use argumentation as a technique to manage disagreement. Indeed, as we have shown elsewhere (Schulz and Rubinelli, 2006), four argumentative action types occur in the medical consultation when doctors justify a diagnosis, justify a treatment, correct patients' wrong behaviours or actions and minimize patients' concerns.

\section{FUNCTIONS OF ARGUMENTATION IN DOCTOR-PATIENT INTERVIEW}

As we shall show in this paragraph, the use of argumentation not only favours doctors' expression of their expertise, but also becomes an effective way of enhancing the quality of the information provided to patients. Again, we will argue that argumentation plays a prominent role for balancing the institutional nature of the relationship between doctors and patients. Here, we are dealing with an aspect of the doctor-patient interaction that, given the current debate on the individualization of an ideal model of interaction, it is crucial to clarify.

\subsection{To give reasons in support of a claim}

In supporting our hypotheses, it is necessary to consider the definition of argumentation in more detail. By drawing on the basic classical explanation of the term, argumentation is the process of giving premises in support of conclusions. To argue means to give reasons for proving the truth of standpoints on issue which are intrinsically problematic and allow different solutions (Van Eemeren and Grootendorst, 2004). As mentioned earlier, medical cases are in essence problematic. By using argumentation, it then becomes clear that doctors can justify their decisions both at the level of diagnosis and prognosis. At the same time, however, while giving reasons they provide patients with the basis for reflecting upon the reasonableness of what is advised. Indeed, when they deliver medical advice in the form of arguments, doctors do not only suggest certain treatments and give indications on the ways these should be followed. They also inform patients on why, for example, certain treatments (and not others) have been prescribed to them specifically, and why these treatments should be followed in certain ways. Argumentation, then, offers information that can be utilized by patients as points of reference for structuring their decisions on the actions to be taken. Insofar as this information is shaped in the format of reasons, the process of argumentation: 
- adds motivators to the internal reasons (factual information and intentional states) and to other propositionally structured entities such as needs, commitments and requirements that the patient might have;

- stimulates the appraisal of the relative weight of the whole set of motivators as a way to arrive at a decision.

\subsection{Argumentation and action}

Another characteristic of argumentation deserves special emphasis. As pointed out earlier, the idea that argumentation prevents patients' freedom, insofar as it strengthens doctors' standpoints and impedes objective decision making, is rather spread. Admittedly, in term of its perlocutionary force, argumentation aims at persuading or convincing someone of the acceptability of a certain standpoint. Yet, even in those cases where argumentations are perceived by people as convincing for their contents and form, this conviction is not enough to motivate actions in the outcome. Just to quote an example, many people do not stop smoking despite several interventions arguing against the side-effects of smoking, and despite the fact that most of those who do not stop smoking believe in the soundness of the current argumentations against this habit. The risk of patronising patients' decisions through argumentation is really low. It becomes even lower in cases where, like in most of doctor-patient interactions, argumentation does not aim to persuade someone of the acceptability of a theoretical claim, but it is aimed at promoting behavioural changes. Indeed, individual decisions in terms of behaviour are ultimately affected by a series of other factors that, as social scientists know well, it is very difficult to determine in its components. Argumentation can surely work as a tool for reducing the gap between the reasons adduced by doctors in support of a certain advice and the actualisation of the decision itself - that is, the patient's actual performance on the advice given. However, it does not impose the speaker's standpoint over an interlocutor. It simply offers the interlocutor reasons to think about its acceptability and to adhere to a certain opinion if $\mathrm{s} / \mathrm{he}$ believes in its reasonableness (Rigotti and Greco, 2005).

In other words, argumentation is not coercive. It rather stimulates in the interlocutor a critical approach toward an issue. ${ }^{6}$ It is probably not an exaggeration to claim that argumentation is actually the only instrument that enables a reasoned compliance of the patient, where the patient takes a certain course of action advised by a doctor because $\mathrm{s} /$ he has understood and believes in the inner motivations behind it. By drawing on one of the fundamental assumptions of the pragma-dialectic theory of argumentation, an argumentative discussion enables parties to deal in a rational way with an issue that allows different solutions. Of course, this becomes particularly relevant when the doctor and the patient explicitly disagree on the 
course of action to be taken. Argumentation can be used "to try to determine to what extent a given standpoint is defensible". In the medical setting, by using argumentation the doctor not only supports his standpoint to the patient, but also exposes the patient to the value of the standpoint itself, enhancing the patient's critical reflection on it. We are here dealing with a constructive dimension of argumentation. This applies both, in a mixed-difference-of-opinion situation, when the patient has already his/her own idea about what should be done to treat his/her disease, and can thus use doctor's advice to make a comparison, refine/correct or further support his/ her standpoint, and in a non-mixed-difference-of-opinion situation, when he/she has no idea and needs to develop one in order to decide on a certain treatment. ${ }^{8}$ Argumentation appears here as a flexible instrument that can be used for different purposes in different contexts. It only presupposes that the speaker who constructs an argument (e.g. the doctor) holds an opinion about a problem (what $\mathrm{s} /$ he thinks the best course of action that the patient should take is), and is willing to present this opinion to the addressee who is more properly a decision maker. ${ }^{9}$ Whether or not the patient is active or passive, and whether $\mathrm{s} /$ he has already his/her own opinion is relevant for the way an argumentative dialogue, in the outcome, might develop, but not for the application of argumentation per se. ${ }^{10}$

\subsection{Argumentation as a balance between doctor and patient}

The last point to consider in discussing the nature and advantages of argumentation in doctor-patient relationship recalls the actual debate on the individuation of an ideal model of discussion between the two parties. In what follows, we shall sketch the main developments of this debate in order to show how argumentation can indeed acquire a prominent role among the instruments for optimising the relationship between doctors and patients.

After the decay of paternalism, where doctors had a prominent and dominant position, today the tendency is to favour interpretations of the doctor-patient relationship that emphasise patients' autonomy: patients are seen as competent to make decisions that concern their own health. ${ }^{11}$ Leaving aside the contract model that, as Walton correctly criticises, cannot be a normative ideal of the doctor-patient relationship, ${ }^{12}$ the reference is first of all to the "informed model", where deliberation and decision making steps are the sole prerogative of the patient. In this context, "information transfer is seen as the key responsibility and only legitimate contribution of the doctor to the decision making process" (Charles et al., 2004, p.781; Quill and Brody, 1996).

In the informed model, the doctor is perceived as a technical specialist who only provides the patient with the relevant information, 
while the patient is free to decide on her/his own. But this role-codification, if it is done in full respect for autonomy and self-determination of the patient, is not unproblematic. As Chin (2002) points out, relegating the role of physician to that of an information-provider plays down the intrinsic inequality of knowledge and skills between doctors and patients. It somehow dismantles the whole nature of doctor-patient relationship that rests on the assumption that the patient seeks assistance from the doctor who is able to provide it. Here it is important to recall what Davis (1997) stresses in his attempt to rebalance exactly this current interpretation of the roles of doctors and patients. Considering the issue from its more philosophical implications, when the patient is ill, s/he has needs that deserve fulfilment. "This", according to the author (1997, p. 178), "is what the physician presents herself as equipped and committed to provide: fulfilment and satisfaction of the ontological need for healing". Accordingly, what is primary to the identity of the physician as health professional is the "central act of profession". Following Pellegrino's interpretation (1979, p. 46), Davies interprets this act by considering the etymological origins of "profession" from the Latin profiteor, meaning "to declare aloud and publicity" and "to promise". And this promise can be articulated in what Pellegrino and Thomasma (1981, p. 48) call the "curative intent" of the physician. ${ }^{13}$

Moreover, it can be added that patients might not be in the position of making choices for their benefit. Considering patients who have only a lay knowledge of medicine, there is a very high possibility that the absence of valuable advice from the doctors' side outweighs the danger that the final choice is made as a result of inappropriate influence (e.g. the influence of people's commonplaces about medical treatments and of unreliable sources available from the internet). The risk is especially high when patients present themselves with a serious or life threatening illness, and when time constraints might not allow wrong decisions in treatments. Indeed, studies show that biophysical and psychosocial factors might highly impact on patients' capacity to make informed choices. ${ }^{14}$

In an attempt to avoid the unbalance within the doctor-patient relationship that the informed model codifies, the literature fosters another scheme of relationship known as the "shared model", where doctors and patients share all stages of the decision making process simultaneously (Charles et al., 2004, p. 781). Doctors and patients express their preferences and end up agreeing on the decisions to be made in "a dialogue rather than an unregulated supplying of information from one party to the other" (Walton, 1985, p. 108). Within this model, doctors do not only provide information but contribute to the decision-making process via negotiation that is, by presenting reasonable settlements that both doctor and patient can live with. An interactive dialogue 
between doctors and patients, according to the forms and models of dialogues that Walton describes in his monographic work, is surely ideal. ${ }^{15}$ However, it encounters some main difficulties that need to be addressed. First of all, given that in the typical medical encounter patients tend to be passive and submissive, ${ }^{16}$ it is not clear within the literature what instruments doctors can use to engage in a dialogue with their patients. Moreover, the "shared model" is not ideal for dealing with clinical situations where treatments do not admit negotiation. To quote an examples, if a patient is not willing to have chemotherapy, while the doctors knows that there is no other option, it is difficult to find a middle term to agree on. Negotiation in term of length and nature of chemotherapy to avoid side effects like hair loss would surely reduce, or even cancel, the potentially beneficial effects of the therapy.

The above limitations support the idea that doctors, without compromising patients' freedom in making the final decision, must be in the position to give advice in order to arouse patients' response and promote a proper negotiation for those cases where negotiation is actually possible. Again, they must be able to express their views whenever the situation is not negotiable, is limited by time constraints or requires clarification.

In this light, a third model of doctor-patient interaction becomes relevant, namely the relationship-centred model discussed by Quill and Brody (1996). This pattern assumes that an open dialogue, in which the physician frankly admits his or her biases, is ultimately a better protector of the patient's right to autonomous choice than artificial neutrality would be. For Quill and Brody, doctors have to offer recommendations in the full service of patients' best interests, and in order to promote an informed autonomy. Doctors' biases should then not be hidden, but should constitute an integral and explicit part of the medical encounter. If this is the case, by considering what we have pointed out in the previous paragraphs, it appears that argumentation is an adequate instrument for the expression of doctors' standpoints. Argumentation can be used to balance an interaction where the doctor performs his/her expert role in front of a patient who seeks expertise in the first place, but who is the only responsible for the final decision to have a certain treatment. By constructing arguments doctors do not patronise the interaction (as they would do if they imposed their biases without supporting them with reasons), but rather they expose their standpoints to be evaluated and pondered by patients.

\section{INVESTIGATING REAL-LIFE ARGUMENTS}

\subsection{The informed consent}

As anticipated in the introduction, the second part of this study is devoted to an exploration of some real-life argumentations. A comprehensive 
analysis of the way argumentations are performed in doctor-patient consultations would surely exceed the limits of the present investigation. However, it is our aim to underline some of the relevant factors to be taken into consideration for basing more systematic research in the field. To conduct our investigation we have recorded a set of 10 doctor-patient consultations in the field of oncology, in collaboration with three Italian physicians. Transcripts of the original consultations have been made by a Master student of the Faculty of Communication Sciences of the University of Lugano, according to a revised version of Jefferson's guidelines (Sacks et al., 1978). In the following sections we shall present a selection of relevant passages translated in English. Original Italian texts are available upon request. ${ }^{17}$ The dialogues recorded run from the moment in which the doctor communicates the diagnosis to the patient, and explains the treatments available, to the signature of the socalled informed consent. In what follows, we shall first discuss briefly the inner constitution of the informed consent and the main difficulties relating to its application. This will help us individuate those aspects of the informed consent which can function as a point of reference for the analysis of the dialogues.

The informed consent is a legal doctrine that has been elaborated by the courts over a number of years. It requires that doctors, before administering medical treatments, elicit the voluntary informed consent of patients. To do so, they are bound to disclose to patients all relevant information about illnesses and treatment options. ${ }^{18}$

The procedure of informed consent has been legalised in order to protect patients by providing them with complete information on which to make an informed decision. In parallel, it is also expected to protect doctors from financial liability provided that the procedure is properly executed. Leaving aside the legal implications of informed consent that we shall not consider in this work, it is generally recognised that this procedure is surrounded by a crucial problem of communication: it is arduous to determine the way of adequately informing patients, and making them understand the information given. In other words, the main question here is: when is the disclosure of information sufficient?

The literature in this area suggests one four approaches concerning the level of disclosure:

1. The reasonable physician standard (or professional standard): this standard allows the physician to determine what information is appropriate to disclose.

2. The reasonable patient standard (or objective standard): it focuses on considering what a patient would need in order to understand the decision at hand.

3. The subjective standard: it requires tailoring information to each patient. 
4. The emergency standard: when valid consent is not possible, the professional standard usually obtains. ${ }^{19}$

All the above approaches show limitations either due to the fact that they are focussed on the physician, rather than the patient, and the former tends to tell the latter very little, or on the evidence that is rather difficult, especially for the limited amount of time that doctors have at their disposal, to tailor medical advice according to patient's individual needs. Guidelines have been given on how information should be generally delivered. These guidelines mainly encourage physicians to avoid a medical terminology and a technical jargon and to encourage patients to ask questions (Lowe and Karridge, 1997).

From the point of view of this paper, there is another important factor to consider. Doctors must give patients enough information on the reasons why they support certain lines of treatments. And this is precisely the aspect on which we shall focus in the following paragraphs. By drawing on some key concepts from argumentation theory, we shall show how doctors' choices of the kind of reasons given in support of advice can impact on the delivery of information that are appropriate for making informed decisions.

\subsection{The analytic method}

In discussing some of the arguments contained in the interactions recorded, we rely on the operations suggested by Van Eemeren and Grootendorst (1992, p.93):

1. Recognizing the points at issue.

2. Recognizing the positions that the parties adopt.

3. Identifying the explicit and implicit arguments.

4. Analysing the argumentation structure.

As far as points 1 and 2 are concerned, in all the arguments selected doctors give their standpoints on the kind of treatment that, according to them, patients should follow. Patients never express a difference of opinion. Yet, the reasons for thinking that in our dialogues doctors perform acts of argumentation, and not simply give information are clear. Next to the treatments suggested there are always possibilities of other treatments (including the non-treatment option). This justifies the presence of argumentative moves which aim at supporting doctors' beliefs in the value of their advice. ${ }^{20}$ As far as point 3 is concerned, we will focus on arguments whose standpoints are explicit, and underline their eventually implicit premises with parentheses. As for point 4, the analysis of the arguments structure will be made by integrating the terminology developed by Van Eemeren et al. (2002, pp. 62-87) with a focus on some relevant aspects of the structures of arguments as codified in classical rhetoric. More specifically, according to classical rhetoricians, 
the efficacy of argumentations was seen as resulting from speakers main abilities: (1) to find the right contents, (2) to dispose them according to the best order, (3) to use an adequate wording. Here the reference is to the abilities presented among others by the anonymous author of Rhetorica ad Herennium in the following passage:

The speaker, then, should possess the faculties of Invention [the Latin inventio], Arrangement [dispositio], Style [elocutio]... Invention is the devising of matter, true or plausible, that would make the case convincing. Arrangement is the ordering and distribution of the matter, making clear the place to which each thing is to be assigned. (Rhetorica ad Herennium, I, 3$)^{21}$

By using the above indications analytically, we intend to test whether doctor's choices at the level of invention, arrangement and wording can indeed suggest some normative elements for an optimisation of their argumentation within the framework of the informed consent.

\subsubsection{On the inventio}

In what follows, three arguments will be analysed.

We read in dialogue 10 :

$04 \mathrm{D}$ : This carcinoma has been already treated. And today

05 visit is due to something different. We have discussed again the

06 situation with the internal radiologists. The decision is that instead of

07 doing the emboli, an embolization again, it is better to do a chemotherapy.

The doctor supports his view that the patient should do chemotherapy, by using a single argumentation from authority that is by claiming that the treatment has been suggested by a team of experts. In a schematic overview:

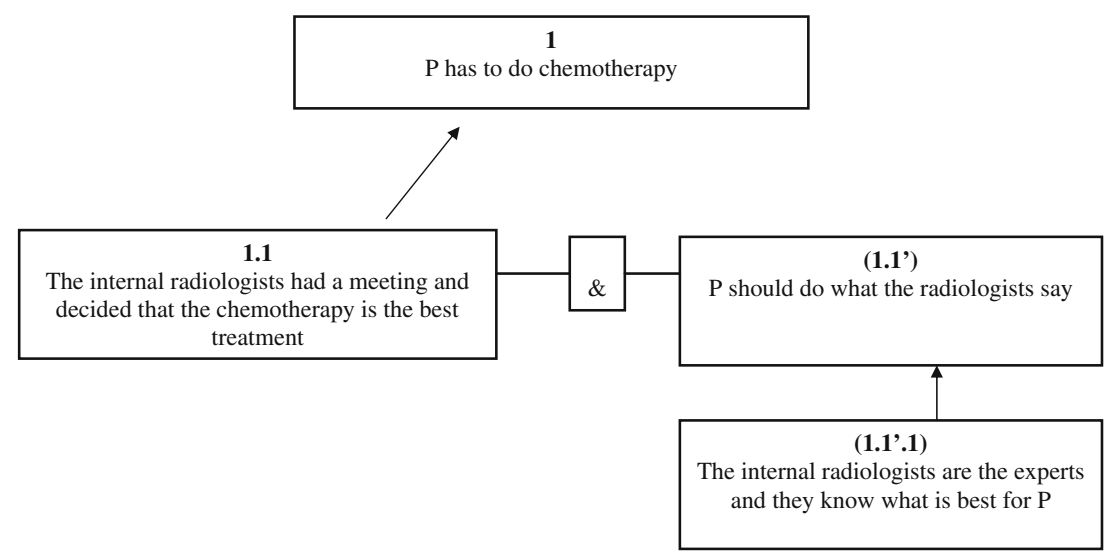


Here the reasons adduced in justification of the standpoint do not reveal any clinical information about the inner motivations for choosing that treatment. Unless the patient has already a correct knowledge of chemotherapy, and is able to understand his/her case by himself, this argument prevents him/her from making any critical reflection oriented to decide or not for the treatment. In the above situation this choice of argument is even more problematic because, we know from other parts of the dialogue, the patient went to the doctor knowing that in a previous consultation a different treatment (namely the embolization) had been suggested. But in the above passage, there is no explanation of why doctors decided to suggest a different clinical action. As a matter of fact, this patient at lines 57-59 of the dialogues interrupts the doctor and asks for an explanation about the change in the treatment:

57 P. I have another question doctor. Why, I mean, before you have 58 decided for the embolization and now for the chemotherapy?

Clearly, if the patient had been passive, he would have missed some essential information.

We read in dialogue 5:

29 D: The exams that you have done while you were at the hospital 30 have revealed that there is a contraction at the esophagus, and 31 there cancer cells have been found. It is for this reason that 32 we must do the therapy.

The scheme of the above coordinative argument runs as follows:

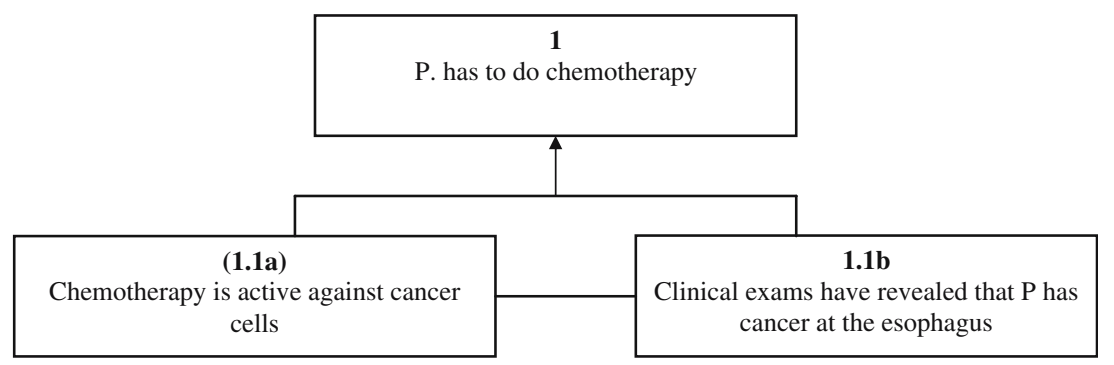

The doctor suggest that the patient should have chemotherapy by giving as reason the clinical evidence that medical tests of the oesophagus have revealed cancerous cells. In comparison with the previous case, in this argument the disclosure of information is higher. However, at the level of inventio the doctor has only partially tailored 
the information given according to the situation of this patient, and has provided him with a general reason that does not necessarily motivate the conclusion. The problem in giving too general information is that the patient may find it difficult to fully understand why this is the best course of action to take for his/her situation. As a matter of fact, having a cancer is not a necessary reason for doing chemotherapy. It is widely known that in some cases of cancer, chemotherapy is not recommended, and surgeries or other kind of less invasive treatments can be taken instead. In this light, a patient who has been given only general information might not understand why s/he should do something that, strictly speaking, could be done differently.

The situation is however different in dialogue 1.

At the beginning of the conversation, the doctor argues in favour of a treatment of radiotherapy. But the problem is that, according to the doctor, radiotherapy is not enough: he would like the patient to start other two treatments, namely the hormonal therapy and the chemotherapy together. To do so, the doctor articulates his argumentation and gives several reasons that deserve to be quoted at length. In the passage, the doctor is presumably aware that the patient might not like a further treatment. In this sense his answer seems to take into account a possible objection by the patient to the addition of the chemotherapy.

$46 \mathrm{D}$ : Our problem now is to control the possibility that some cells have spread around. By analysing the pure biological characteristics of this tumour have seen that it reacts to hormonal receptors. Thus, you could only do this hormonal therapy. The hormonal therapy is a pill, called tamoxifene, that in practice interacts with the receptors of hormones in order to stop the spread of these cancer cells. It is a pill that normally you take for five years. It is useful for the whole body and it is also useful to protect your breast from eventual other tumours.

56 In fact, if one has had a cancer somewhere,

57 the person has more possibilities to have a breast cancer

58 than the person who did not have a contralateral cancer.

59 In this case, a pill would also partly protects you from this

60 possibility. Going back to what you should do,

61 this is surely a radiotherapy and

62 surely the hormonal therapy.

63 Now we have to decide whether or not we should

64 also add this bloody chemotherapy. Without any doubts at your age, maybe in 
65 worse conditions, we would be happy to give only the pill. Speaking

66 frankly, the addition of chemotherapy in this situation would

67 increase the possibility of healing.

68 Considering your situation, your age, the lymph nodes and

69 your general state this imply having a 6-7\% further possibilities

70 to heal. This is really the main point of the issue

$\cdots$

81 By doing the hormonal therapy we diminish both the possibility

82 of developing another cancer in another part of the body and

83 the possibility that your illness appears again about $10 \%$.

84 If we also add the chemotherapy we add another $6-7 \%$

85 of possibility of healing...

The structure of the above complex argument is the following:

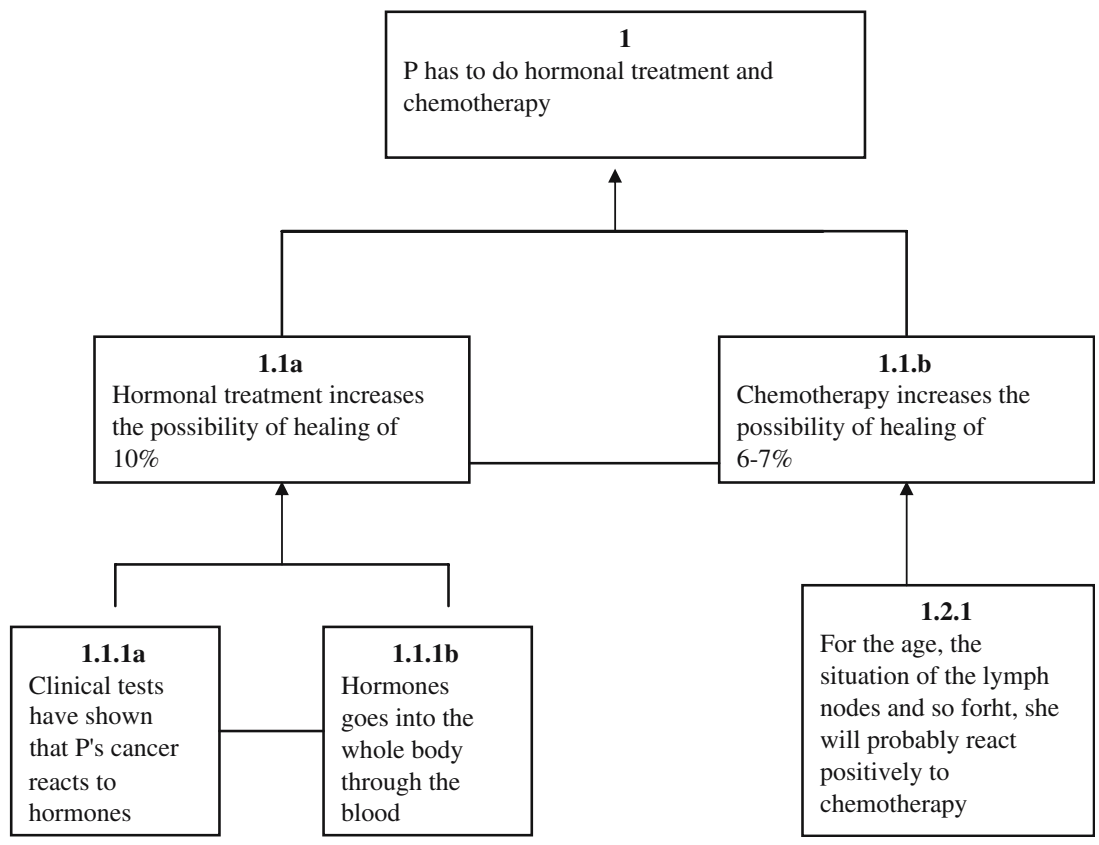

The relevant aspect concerning the reasons given in the above argument is that the doctor is not simply saying that, because the patient has been diagnosed with cancerous cells, she should undertake this course of action, like in the previous case. But he motivates the conclusion by illustrating the advantages of the two treatments proposed as inserted in the clinical situation of this patient. The doctor explains in detail what the treatments suggested are, and how they would work in the context of this patient. We could say that the reasons for 
suggesting a treatment have been tailored in accordance with the specific characteristics of the patient.

\subsubsection{On the dispositio}

In considering the arrangement of the contents that doctors use in arguing their standpoint, we shall pay attention to the relationship between the order of presentation of the contents and the disclosure of information required by patients to make critical decisions about treatments. Here, a main factor arises from our analysis. As we have seen at paragraph 4.1, the procedure of informed consent requires physicians to discuss with patients, among other things, the treatment suggested, its nature and side effects. But in the dialogues observed, we noted that doctors tend to arrange these contents as if they were separated one from the other, and had no logical connection. More specifically, there are cases where a doctor suggests a treatment and argues in favour of it without however explaining what the treatment is. The explanation only comes in a different stage of the consultation. As the following case shows, this separation might de-optimise the exchange of information.

In dialogue 8, at lines $132 \mathrm{ff}$. the doctor mentions the patient different treatments and suggests chemotherapy:

132 D: You did the bronchoscopy. They aspirated some catarrh. There

133 was a big inflammation.

134 But some cells were of tumoral origin, and you did surely

135 understand this...

136 P: Of course.

137 D: Nowadays we have more treatments, and these are of

138 different kinds. They are chemotherapeutic, radiotherapeutic

139 and sometimes surgical.

140 Today there are some other treatments that are not

141 chemotherapeutic, but that we cannot do at this stage.

142 Thus, while the illness is on, the first approach is surely to fight

141 the cancer with a chemotherapy. kind:

The doctor develops a coordinative argumentation of the following 


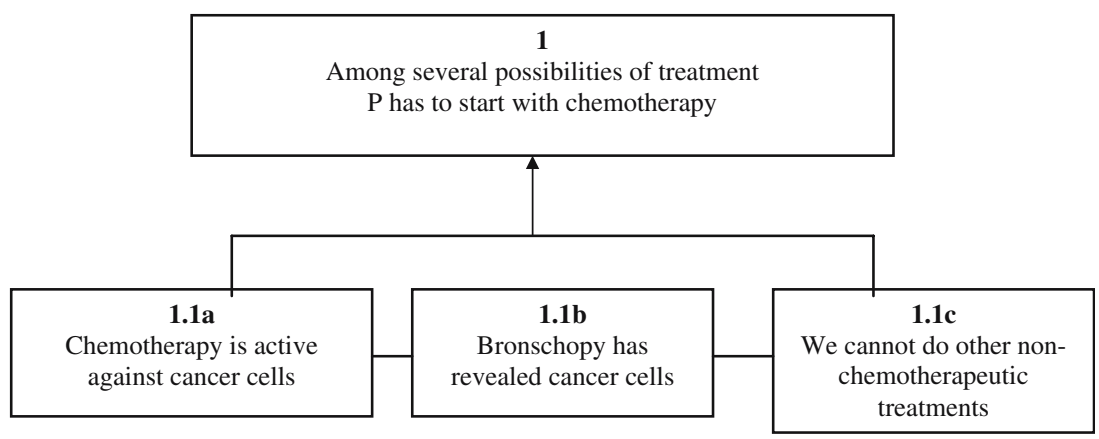

From the perspective of this paper, the above argument has three main limitations. Firstly, the reasons adduced by the doctor are of the similar kind to those analysed in the second argument of the previous paragraph. Secondly, the doctor does not provide reasons why the patient should do chemotherapy instead of the other treatments mentioned. In particular, he does not give reasons to support the unfeasibility of non-chemotherapeutic treatments. Thirdly, and we enter into the domain of dispositio, the doctor does not explain at this stage what all the treatments is talking about are, and not even what the chemotherapy that he would like the patient to start is. This omission is even more problematic because several lines later, after having visited the patient, the doctor recalls his standpoint - that the patient should do chemotherapy - and even mentions the possibility of eventually adding radiotherapy:

259 D: Surely, in theory, we should do chemotherapy. Eventually

260 this could be associated with radiotherapy.

261 I mean the mix of chemo and radio

262 could be done. It is a bit more toxic,

263 but we do it if we think that it is right

264 to do it in a second time. It is

265 better to do only chemotherapy at the beginning.

266 We shall see in a second time

267 if it is possible to use medicaments in a higher dose.

Only after having proposed the treatments, the doctor asks the patient if he knows what chemotherapy and radiotherapy is. And the answer is negative:

265 D: Do you know what chemo and what radiotherapy are?

266 P: Could you explain it?

Thus, at lines $267-315$, the doctor gives then a very general explanation of what these two treatments are. Now suggesting a treatment 
without explaining from the start, what it is and how it works might prevent the patient from understanding why it is actually been advised. More specifically, the problem of giving a general and separate description of the treatment is that the patient must then infer by him/herself how this treatment would work in his/her case. As already mentioned, this can be problematic since, in the majority of cases, the patient is not an expert in the field. Indeed, in dialogue 7, the doctor argues in favour of a treatment composed both of chemotherapy and radiotherapy by mentioning the possibility of success, but without explaining what they are. At lines 114 the patient interrupts the doctor and asks for this explanation, as this information was crucial to understand the doctor's position:

110 D: A way of increasing the possibility of success would be to do this

111 chemotherapeutic treatment first and then, after a few cycles, some

112 radiotherapy more or less associated with some

113 more chemotherapy depending...

114 P: Chemotherapy? What is it?

Here, again, we can wonder about the quality of this patient's decision making, if he had not been active enough to ask for an explanation of the treatment.

\subsubsection{On the elocutio}

The literature on the modalities of wording health messages in doctor-patient interaction is vast. A lot has been written on the wording of the questions that doctors ask patients, on the use of metaphors and other rhetorical devices and so forth. ${ }^{22}$ In the framework of this paper, however, we want to focus our attention on some of the words used by physicians while declaring their standpoints. Our investigation stresses that physicians must show the reasons behind their standpoints, and enable patients to think about the choice to be made. As will be shown, there are cases where the use of certain modal verbs and other linguistic expressions seem to play down the fact that it is the patient who has to make the final decision, and that no treatment suggested by physicians is strictly speaking necessary.

In dialogue 1 , the doctor tells the patient that he should have chemotherapy because this increases the possibility of healing by $6 / 7 \%$. Yet, before starting to explain what kind of chemotherapy he is thinking about, he remarks that the there is still a decision to be made before proceeding with the actual treatment:

81 D: By doing the hormonal therapy we diminish of about $10 \%$ both

82 the possibility to develop another cancer

83 in another part of the body and 
84 the possibility that your illness appears again. If we also add the

85 chemotherapy we add another $6-7 \%$ of possibility of healing.

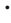

87 The kind of chemotherapeutic treatment if you and I decide to do it

88 will be a mix of two medicaments

The same attitude is recognisable in a passage of dialogue 10, where the doctors uses the expression "we are thinking" clearly underlining that the suggestion he gives only represents the medical point of view:

$22 \mathrm{D}$ : We are thinking about doing a chemotherapy that consists

23 in giving two medicaments...

Again, in dialogue 9 the doctor specifies that what he advises is simply a proposal:

$50 \mathrm{D}$ : This is then the main point. It has been confirmed during

51 the surgery that it is a malignant tumour...

...

64 Thus, in these situations, we always propose a treatment based

65 on chemotherapy that we call adjuvant

On the contrary, in the following case the doctor uses the modal verb "must" that, for its strength, could give the patient the impression that this is the necessary and only possible option:

Dialogue 5

$30 \mathrm{D}$ : There is this contraction at the esophagus

31 and there we have found tumorous cells

32 It is for this reason* that we must do the chemotherapy.

It might be the case that in terms of treatments available this is the only applicable, or the only solution that could be beneficial. Yet, an imperative wording like this is in contrast with giving the patient the option of non-treatment, even if this last choice is in contrast with doctor's point of view.

Finally, we have noted how in some of the interactions recorded doctors argue in favour of their standpoints by also introducing percentages of the possibility of healing. Instead of simply saying that a treatment has beneficial effects, and show what these are, they also try to quantify the benefit itself. Percentages, on the one hand, accentuate the precariousness of the nexus treatments-expected result in medical issues, on the other they hand can give patients more objectivity in pondering a certain decision, as well as a concrete datum on which they can base the 
decisions themselves. The passages in questions read as follows. In dialogue 9 , the doctor, while arguing in favour of chemotherapy, remarks:

84 D: The chemotherapy does not protect you completely from having

85 a relapse. These are things that you should know.

86 It reduces of about $15 \%$ the possibility

87 that your illness appears again there or in another part.

88 There is a high possibility that you're already fine. We can further

89 increment this possibility by doing chemotherapy.

90 Unfortunately, in medicine we cannot assure $100 \%$ results.

Again, in dialogue 1, the doctor argues in favour of a mixed treatment and inserts percentages about its benefits:

78 D: Unfortunately, in medicine we cannot be $100 \%$ sure

79 that we get maximum results.

80 We say that by doing the radiotherapy we diminish

81 the possibility of a local relapse.

82 If we do the hormonal therapy we diminish of about $10 \%$

83 both the possibility of having another tumour

84 in another part of the body and the relapse of the illness.

85 By adding the chemotherapy we add another $6-7 \%$

86 of possibilities of healing.

\section{CONCLUSION}

Throughout this article we have been considering the nature and impact of argumentation in doctor-patient interaction. One of the most important theses of our analysis is that argumentation should be considered as a powerful instrument for the performance of a balanced relationship between doctors and patients. Another important conclusion of our analysis is that a reflection on doctors' choices at the level of the selection, arrangement and style of arguments can highly impact on the transfer of information that are relevant for enhancing patients' decision making. In the dialogues observed, we have noted how generic reasons, as well as a separation of topics that are logically connected, can fail to provide crucial information for understanding doctors' standpoints. Again, the wording of doctors' advice can create an imbalance in the doctor-patient relationship, insofar as it can prevent patients from considering possibilities of treatments other than those suggested by their doctors during the medical encounter. We have stressed on more than one occasion, that our investigation is only exploratory. More systematic studies based on a wider corpus of dialogues are surely needed. A canon of normative advice for enhancing a 
critical discussion between doctors and patients should be created. This would presuppose a deeper and more structured understanding of the argumentative manoeuvrings that occur between doctors and patients. In particular it should be determined whether different argumentation strategies make a difference in patients' response in term of adherence. Again, a possible line of investigation would entail exploring what counts as reasonable or unreasonable argument within this context. This last point, in particular, would then motivate further research on the use and impact of fallacies in the medical setting. Finally, more analyses are needed on the nature of the contents that doctors uses in their arguments and, in particular, on the shift between emotional versus medical contents. To cut a long story short, there seems to be a lot at stake in the field! But at the stage of the present analysis, if we have succeeded in giving a challenging glimpse into the richness of argumentation in the medical consultation, then we will have achieved our aim.

\section{ACKNOWLEDGEMENTS}

The authors wish to thank for the several precious discussions on sections of this paper Prof. Eddo Rigotti, Professor Franz van Eemeren, Professor Peter Houtlosser, Dr. Agnès Van Rees and the other colleagues who attend the annual Amsterdam-Lugano colloquium. Special thanks also go to the anonymous reviewers who provided very detailed and constructive comments.

\section{NOTES}

1 See Plato's Gorgias 456 A, where the philosopher asserts through the characters of his master Socrates and the sophist Gorgias that doctors and orators should work together.

2 See in particular Van Eemeren and Grootendorst (2004).

3 Thus, Grasso et al. (2000) 53 attempt to apply the New Rhetoric's system of argumentation to the domain of health promotion, without fully exploring what argumentation actually is there. The authors assume a distinction between beliefs and opinions which does not seem to be altogether clear. Beliefs appear among the material causes of opinions. Bachers et al., (2002) focus on the argumentative burdens of self-advocacy. This perspective, although interesting, needs to be implemented by an analysis of the argumentative features and potentialities of the doctor's role. It is in fact generally recognised that in the typical physician-patient interaction doctors are dominant while patients are submissive. See, for example, BallardReisch (1990) and Frederikson (1993). Upshur and Colak (2003) focus almost exclusively on the role and use of evidence-based approaches to support conclusions and recommendations. Finally, Dickinson (1998) uses a model of argumentation to make explicit the nature and function of data in decision-making. In his paper, however, it is not clear where and in what context he sees argumentation. The ambiguity of his approach seems to derive from the fact that he considers the term argumentation in the wide and general sense of reasoning. But, although argumentation is indeed a process of reasoning, not every process of reasoning is argumentation. 
4 Evidence-based Medicine working group (1994) and Upshur and Colak (2003, p. 283).

5 See in particular Schulz (2003).

6 On this point see in particular Perelman and Olbrechts-Tyteca (1958, Ch. 1).

7 Van Eemeren et al. (2002, p. 24).

8 On the implicit/explicit difference of opinion see, more specifically, Schulz and Rubinelli (2006, pp. 88-92).

9 See Aristotle's on the concept of krités as underlined by Rigotti and Greco (2005).

10 On this point see more in Van Eemeren et al. (2002, pp. 228-29).

11 See for example Eddy (1990), Laine and Davidoff (1996) and Tan NHSS (2002) with further references.

12 As Walton (1985, p. 8) remarks: "...a contract...requires exchange of "clearly defined commodities or benefits. The patient hands over his fee (directly or indirectly, depending on medical funding practices of the locality), but what commodity does the doctor produce in exchange? The premises of fallibility of treatment outcomes suggests that the doctors may be unwise, even foolish, to contract for specific outcome in advance".

13 See Davis (1997, p. 178).

14 See Chin (2002), Wear (1998), Pellegrino (1979) and Beauchamp (1994).

15 See in particular Walton's chapter on practical reasoning (1985, pp. 69-98).

16 See Ballard-Reisch (1990) and Frederikson (1993).

17 For requesting the original quotations, please send an email to sara.rubinelli@lu.unisi.ch.

18 For a full analysis of the structure of the informed consent see Faden and Beauchamp (1986) and Doyal (2000).

19 For a full treatment of this topic see Applebaum et al. (1987).

20 On the difference between explanation and argumentation see Henkemans (2001).

21 In classical rhetoric, the officia oratoris are actually five. To the three already mentioned the author also quotes "memory" that is the firm retention in the mind of the matter, words, and arrangement, and "delivery" that is the graceful regulation of voice, countenance, and gesture. The concepts of memory and delivery will be however left out of our analysis. Memory becomes in fact relevant only when speeches are long, prepared in advance and presented without any technological support. Delivery becomes a pertinent category in considering the emphatic aspects of the dialogues that, however, we do not intend to analyse within our perspective.

${ }^{22}$ For a review specifically focussed on the oncological setting see Bredart et al. (2005). See also Maguire and Pitceathly (2002) and Caffi (2002).

\section{REFERENCES}

Applebaum, P. S., C. W. Lidz and A. Meisel: 1987, Informed Consent: Legal Theory and Clinical Practice, Oxford University Press, New York, NY.

Bachers, D. E., S. M. Haas and L. J. Neidig: 2002, 'Satisfying the Argumentative Requirements for Self-advocacy', in Van Eemeren, op. cit., pp. 291-308.

Ballard-Reisch, D. S.: 1990, A Model of Participative Decision Making for Physician-Patient Interaction, Health Communication 2, 91-104

Beauchamp, T. L.: 1994, Childress JF. Principles of Biomedical Ethics, 4 ed.Oxford University Press, New York.

Bredart, A., C. Bouleuc and S. Dolbeault: 2005, Doctor-Patient Communication and Satisfaction with Care in Oncology, Current Opinion in Oncology 17(4) 351-354.

Caffi, C.: 2002, La Mitigazione. Un approccio pragmatico alla comunicazione nei contesti terapeutici, Lit Verlag, Münster.

Charles, C., T. Whelan and A. Gafni: 2004, What Do We Mean by Partnership in Making Decisions About Treatment?, British Medical Journal 319(7212) 780-782. 
Chin, J. J.: 2002, Doctor-Patient Relationship: From Medical Paternalism to Enhanced Autonomy, Singapore Medical Journal 43(3) 152-155

Davis, D. D.: 1997, Phronesis, Clinical Reasoning, and Pellegrino's Philosophy of Medicine, Theoretical Medicine 18(1-2) 173-195

Dickinson, H. D.: 1998, Evidence-based Decision-making: An Argumentative Approach, International Journal of Medical Informatics 51(2-3) 71-81

L. T. Doyal, (ed.): 2000, Informed Consent in Medical Research, BMJ Publications, London.

Eddy, D. M.: 1990, Clinical Decision Making: From Theory to Practice Anatomy of Decision, The Journal of the American Medical Association 263(3) 441-443.

Evidence-based Medicine Working Group: 1994, Evidence-based Medicine: A New Approach to Teaching the Practice of Medicine, Journal of Dental Education 58(8) 648-653.

Faden, R. and T. Beauchamp: 1986, A History and Theory of Informed Consent, Oxford University Press, New York.

Frederikson, L. G.: 1993, Development of an Integrative Model for Medical Consultation, Health Communication 5, 225-237

Grasso, A., A. Cawsey and R. Jones: 2000, Dialectical Argumentation to Solve Conflicts in Advice Giving: A Case Study in the Promotion of Healthy Nutrition, International Journal of Human-Computer Studies 53(6) 1077-1115.

Henkemans, F. S.: 2001, 'Argumentation, Explanation and Causality: An Exploration of Current Linguistic Approaches to Textual Relations', in T. Sanders and W. Spooren (eds.), Text Representation. Linguistic and Psycholinguistic Aspects, pp. 231-247, John Benjamins Publishing Company.

Laine, C. and F. Davidoff: 1996, Patient-centered Medicine. A Professional Evolution, The Journal of the American Medical Association 275(2) 152-156.

Lowe, M. and I. Kerridge: 1997, Informed Consent and Shared Decision Making, Student British Medical Journal 5, 275-277.

Maguire, P. and C. Pitceathly: 2002, Key Communication Skills and How to Acquire Them, British Medical Journal 325(7366) 697-700.

Pellegrino, E. D.: 1979, Toward a Reconstruction of Medical Morality: The Primacy of the Act of Profession and the Fact of Illness, Journal of Medicine and Philosophy 4(1) $32-56$

Pellegrino, E. D. and D. C. Thomasma: 1981, A Philosophical Basis of Medical Practice: Toward a Philosophy and Ethic of Healing Professions, Oxford University Press, New York.

Perelman, C. and L. Olbrechts-Tyteca: 1958, Traité de l'Argumentation. La Nouvelle Rhétorique, Presses Universitaires de France.

Quill, T. E. and H. Brody: 1996, Physician Recommendations and Patient. Autonomy Finding a Balance Between Physician Power and Patient Choice, Annals of Internal Medicine 125(9) 736-769.

Rigotti, E. and S. Greco: 2005: 'Argomentazione nelle istituzioni' ARGUMENTUM eLearning module, www.argumentum.ch.

Sacks, H., E. Schegloff and G. Jefferson: 1978, A Simplex Systematic for the Organization of Turn-Taking for Conversation, Language 50(4) 696-735.

Schulz, P.J.: 2003, 'Effetti mediatici sull'interazione medico-paziente', in S. Rubinelli and B. Crivelli (eds.), Televisione, stampa e internet tra medico e paziente, Tribuna Medica, 7-10 (Special Issue).

Schulz, P.J.: 2006, 'The Communication of Diagnostic Information by Doctors to Patients in the Consultation', in P. Twohig and V. Kalitzkus (eds.), Bordering Biomedicine, pp. 103-118.

Schulz, P.J. and S. Rubinelli: 2006, 'Healthy Arguments for Literacy in Health', Proceedings of the American Association for Artificial Intelligence (AAAI). Spring Sym- 
posium on Argumentation for Consumers of Healthcare, Palo Alto, CA, March 2006, pp. 86-95.

Tan, NHSS: 2002, Deconstructing Paternalism - What Serves the Patient Best?, Singapore Medical Journal 43(3) 148-151

Upshur, R.E.G. and E. Colak: 2003, Argumentation and Evidence, Theoretical Medicine and Bioethics 24(4) 283-299.

Eemeren, F. and R. Grootendorst: 1992, Argumentation, Communication, and Fallacies. A Pragma-Dialectical Perspective, Lawrence Erlbaum Associates Publishers, Hillsdale and London.

Eemeren, F., R. Grootendorst and F. S. Henkemans: 2002, Argumentation. Analysis, Evaluation, Presentation, Lawrence Erlbaum Associates Publishers, Mahwah and London.

Eemerenm, F. and R. Grotendorst: 2004, A Systematic Theory of Argumentation, The pragma-dialectical approach, Cambridge.

Walton, D. N.: 1985, Physician-Patient Decision-making. A Study in Medical Ethics, Greenwood Press, Connecticut and London.

Wear, S.: 1998, Informed Consent: Patient Autonomy and Clinician Beneficence Within Health Care, Georgetown University Press, Washington, DC. 